\title{
Approaches for Reduced Order Modeling of Electrically Actuated von-Karman Microplates
}

\author{
Shahid Saghir \\ King Abdullah University of Science and Technology (KAUST) \\ 4700 KAUST, Thuwal 23955-6900, Saudi Arabia \\ shahid.saghir@kaust.edu.sa
}

\author{
M.I. Younis ${ }^{1}$ \\ King Abdullah University of Science and Technology (KAUST) \\ 4700 KAUST, Thuwal 23955-6900, Saudi Arabia \\ mohammad.younis@kaust.edu.sa
}

\begin{abstract}
This article presents and compares different approaches to develop reduced order models for the nonlinear vonKarman rectangular microplates actuated by nonlinear electrostatic forces. The reduced-order models aim to investigate the static and dynamic behavior of the plate under small and large actuation forces. A fully clamped microplate is considered. Different types of basis functions are used in conjunction with the Galerkin method to discretize the governing equations. First we investigate the convergence with the number of modes retained in the model. Then for validation purpose, a comparison of the static results is made with the results calculated by a nonlinear finite element model. The linear eigenvalue problem for the plate under the electrostatic force is solved for a wide range of voltages up to pull-in. Results among the various reduced-order modes are compared and are also validated by comparing to results of the finite-element model. Further, the reduced order models are employed to capture the forced dynamic response of the microplate under small and large vibration amplitudes. Comparison of the different approaches are made for this case.
\end{abstract}

Keywords: electrically actuated microplates, static analysis, dynamics of microplates, diaphragm vibration, large amplitude vibrations, nonlinear dynamics 


\section{INTRODUCTION}

Reduced order models (ROMs) have been shown to be time efficient and powerful tools to investigate the static and dynamic behavior of Micro-Electro-Mechanical Systems (MEMS) devices [1-6]. Modeling of MEMS devices poses several challenges, such as inherent nonlinearities and microscale instabilities. Nonlinearities include geometric nonlinearities, the nonlinear parallel-plate electrostatic force, and squeeze film damping [7]. Most of the MEMS modeling works can be categorized into two classes. In the first class, the underlying structures are assumed to behave linearly, and hence a linear plate or beam theory are used to model them [8-14]. In the second class, the geometric nonlinearity is accounted for via nonlinear plate theories, such as von-Karman or Mindlin theories [1, 3, 15-17]. Recently, modified couple stress theory has been used to model the size dependent behavior of microplates $[18,19]$.

The solution methods reported in literature include Finite Element Method (FEM) [15, 20-22], Boundary Element Method (BEM), Differential Quadrature Method (DQM) [23-25], and Runge Kutta method. FEM based software tools are computationally expensive and may not be suitable for complicated nonlinear dynamic analysis. Reduced order models on the other hand, based on the Galerkin method, are computationally efficient and are capable to perform parametric studies to reveal the effect of different design parameters [1-3]. Also they can be implemented in sophisticated nonlinear dynamics tools, such as shooting.

Among the previous works on microplates, Machauf et al. [8] studied the characteristics of an electrostatically actuated micropump. They used the linear plate theory to model the mechanical motion of the pump diaphragm. A ROM was used to predict the performance of the pump. Chao and co-workers [9] used the linear plate theory to model the fully clamped thin 
plate under electrostatic pressure. They employed a reduced order model to develop an analytical expression for the pull in voltage of the flexible thin plate in the applications of microphone and switches. Nayfeh and Younis [10] used the linear plate theory to model squeeze film damping in microplates. A compressible Reynolds's equation was used to model the squeeze film damping effect. A combination of perturbation and FEM was used to solve for the structural modeshapes, the pressure distribution, the natural frequencies, and the quality factors. Theoretically calculated quality factors were found in good agreement with the experimental data. Bertarelli et al. [11] investigated a circular diaphragm micropump under electric actuation using a linear plate theory and a FE model. They analyzed the behavior of the micropump under quasi static and dynamic electric loading. Ahmad and Pratap [12] investigated the static response of a clamped circular plate under electrostatic load using the Galerkin method. Porfiri [13] investigated the small vibrations of a parallel array of identical microplates deflected under electric loading. He showed that the vibrational properties can be tuned by properly selecting the DC voltage across the adjacent microplates. Srinivas [14] investigated the static and dynamic pull-in of simply supported microplates using a closed form solution and compared the results with those of a Galerkin approximation.

$\mathrm{Ng}$ et al. [15] performed dynamic analysis of microplates under electrostatic forces. They used the BEM to solve the Laplace equation for the electric potential to calculate the charge density and the corresponding electric force. The first order shear deformation theory (FSDT) was used to model the plate motion and FEM was utilized to discretize the governing equations.

Mukherjee et al. [26] and Telukunta et al. [22] used a fully Lagrangian approach to analyze the coupled electro-mechanical field of a MEMS microplate. They employed FEM for the analysis of 
the mechanical deformations in the plate and the BEM to obtain the electric field exterior to the plate.

Vogl and Nayfeh [1] presented an analytical ROM based on the Galerkin method for fully clamped electrostatically actuated circular plates. The model accounted for the geometric nonlinearity and residual stresses. Faris et al [27] presented a model for a micropump based on electrostatically actuated annular plates. The model predicts the deflection accurately for any voltage up to the pull-in voltage. Mohammadi et al. [25] investigated the pull-in instability of electrostatically actuated circular microplates. They used the strain gradient elasticity theory to account for the size effects. A generalized differential quadrature method (GDQ) was used to solve the governing differential equations. Zhao et al. [3] presented a ROM model based on the Galerkin method for electrostatically actuated rectangular microplates. The model accounts for the nonlinearities due to electric force and mid-plane stretching through the von-Karman strains. They investigated the static deflection under the applied DC voltage. Natural frequencies and mode shapes were calculated around the deflected position. Zand and Ahmadian $[20,28]$ investigated the pull-in and vibrational behavior of single and multilayer microplates under electric actuation and squeeze film damping. They used a combination of FEM and finite difference method (FDM) to solve the system of equations.

Fu and Zhang [29] investigated the active control of the nonlinear static and dynamic responses of piezoelectric viscoelastic microplates actuated electrically. They employed the nonlinear vonKarman equations of the plate and used the Galerkin method to discretize the equations. Karimzade et al. [30] studied the nonlinear pull-in instability of a fully clamped microplate with 
movable base. They solved the governing equation using the extended Kantorovich method and the Galerkin approximation technique.

In recent years, the modified couple stress theory has attracted several researchers for nonlinear analysis of micro structures [18, 19, 31-33]. Gholipour et al. [19] investigated the inplane and out-of-plane size dependent nonlinear dynamics of microplates resting on elastic foundation. Farokhi and Ghayesh [31] investigated the dynamic behavior of geometrically imperfect microplates.

Despite the aforementioned several works on the mechanical behavior of microplates; few report ROM for investigation of the mechanical behavior of the nonlinear von-Karman rectangular microplates actuated electrically. These studies are limited to the static and linear vibrations. Nonlinear vibrations under large electrostatic exciting forces has not been reported in the literature. Hence, there is a need to develop ROMs that enable investigating the nonlinear behavior of microplates under large electrostatic loading and while undergoing large deflections.

In this article we present different approaches to develop a ROM for the nonlinear von-Karman rectangular microplates to investigate the static and dynamic behavior under small and large actuating forces. Small forces refer to the forces, which are sufficiently small that does not activate the nonlinear behavior, and hence the plate response is linear. Large forces on the other hand lead to large deflections of the plate that are comparable with the thickness of the plate; and hence activates the plate geometric nonlinearities. In this study we consider a fully clamped microplate. The model accounts for the geometric nonlinearity as well as the 
nonlinearity due to the electrostatic force. Different types of basis functions are used in conjunction with the Galerkin method to discretize the governing equations.

\section{PROBLEM FORMULATION}

In this study we consider a fully clamped rectangular microplate (Fig. 1) and adopt the dynamic version of the von-Karman equations to describe the plate motion [34, 35]. We ignore the inplane inertia since the in-plane natural frequencies are much higher than the transverse natural frequencies. Hence, the in-plane inertia has negligible effect on the transverse motion.

Accordingly, the governing equations can be written as

$$
\begin{aligned}
& \frac{\partial^{2} u}{\partial x^{2}}+\frac{1}{2}(1+v) \frac{\partial^{2} v}{\partial x \partial y}+\frac{1}{2}(1-v) \frac{\partial^{2} u}{\partial y^{2}}+\frac{\partial w}{\partial x} \frac{\partial^{2} w}{\partial x^{2}}+\frac{1}{2}(1+v) \frac{\partial w}{\partial y} \frac{\partial^{2} w}{\partial x \partial y}+\frac{1}{2}(1-v) \frac{\partial w}{\partial x} \frac{\partial^{2} w}{\partial y^{2}}=0 \\
& \frac{\partial^{2} v}{\partial y^{2}}+\frac{1}{2}(1+v) \frac{\partial^{2} u}{\partial x \partial y}+\frac{1}{2}(1-v) \frac{\partial^{2} v}{\partial x^{2}}+\frac{\partial w}{\partial y} \frac{\partial^{2} w}{\partial y^{2}}+\frac{1}{2}(1+v) \frac{\partial w}{\partial x} \frac{\partial^{2} w}{\partial x \partial y}+\frac{1}{2}(1-v) \frac{\partial w}{\partial y} \frac{\partial^{2} w}{\partial x^{2}}=0 \\
& \frac{h^{2}}{12} \nabla^{4} w+\frac{\rho\left(1-v^{2}\right)}{E h} \frac{\partial w}{\partial t^{2}}+\frac{c\left(1-v^{2}\right)}{E h} \frac{\partial w}{\partial t}=\frac{\varepsilon\left(1-v^{2}\right) V(t)^{2}}{2 E h(d-w)^{2}}+\frac{\left(1-v^{2}\right)}{E h}\left(N_{x x} \frac{\partial^{2} w}{\partial x^{2}}+2 N_{x y} \frac{\partial^{2} w}{\partial x \partial y}+N_{y y} \frac{\partial^{2} w}{\partial y^{2}}\right) \\
& +\frac{\partial u}{\partial x}\left(\frac{\partial^{2} w}{\partial x^{2}}+v \frac{\partial^{2} w}{\partial y^{2}}\right)+\frac{\partial v}{\partial y}\left(v \frac{\partial^{2} w}{\partial x^{2}}+\frac{\partial^{2} w}{\partial y^{2}}\right)+(1-v)\left(\frac{\partial u}{\partial y}+\frac{\partial v}{\partial x}\right) \frac{\partial^{2} w}{\partial x \partial y} \\
& +\frac{1}{2}\left(\frac{\partial w}{\partial x}\right)^{2}\left(\frac{\partial^{2} w}{\partial x^{2}}+v \frac{\partial^{2} w}{\partial y^{2}}\right)+\frac{1}{2}\left(\frac{\partial w}{\partial y}\right)^{2}\left(v \frac{\partial^{2} w}{\partial x^{2}}+\frac{\partial^{2} w}{\partial y^{2}}\right)+(1-v)\left(\frac{\partial w}{\partial x} \frac{\partial w}{\partial y} \frac{\partial^{2} w}{\partial x \partial y}\right)
\end{aligned}
$$

where $u, v$ and $w$ are the displacements along $x, y$ and $z$ direction, respectively, $\nabla^{4}$ is the bi-

harmonic operator given by; $\nabla^{4}=\frac{\partial^{4}}{\partial x^{4}}+2 \frac{\partial^{4}}{\partial x^{2} \partial y^{2}}+\frac{\partial^{4}}{\partial y^{4}}, h$ is the plate thickness, $d$ is the parallel plate capacitor gap, $\rho$ is the mass density, $c$ is the damping coefficient, and $v$ and $E$ are Poisson's ratio and modulus of elasticity, respectively, $V(t)$ is the applied voltage; it is either $V_{d c}$ for static analysis or a $V_{a c}$ superimposed to $V_{d c}$ i.e. $V(\mathrm{t})=V_{d c}+V_{a c} \operatorname{Sin}(\Omega \mathrm{t})$, where $\Omega$ is the 
actuating frequency. The first term on the right side of (3) i.e. $\frac{\varepsilon\left(1-v^{2}\right) V(t)^{2}}{2 E h(d-w)^{2}}$ is the applied electric pressure in the transverse direction while $N_{i j}$ are the inplane membrane forces, applied on the $i^{\text {th }}$ edge in the $j^{\text {th }}$ direction such forces may appear in the microplates during the micro fabrication process. It is worth to mention here that the inplane equations (1) and (2) coupled with nonlinear terms on the right hand side of equation (3) represent the contribution due to mid-plane stretching.

The associated boundary conditions are

$$
\begin{gathered}
u(0, y, t)=v(0, y, t)=u(a, y, t)=v(a, y, t)=0 \\
u(0, y, t)=v(0, y, t)=u(b, y, t)=v(b, y, t)=0 \\
w(0, y, t)=w(a, y, t)=0 \\
\frac{\partial w}{\partial x}(0, y, t)=\frac{\partial w}{\partial x}(a, y, t)=0 \\
w(x, 0, t)=w(x, b, t)=0 \\
\frac{\partial w}{\partial y}(x, 0, t)=\frac{\partial w}{\partial y}(x, b, t)=0
\end{gathered}
$$

For convenience, we introduce the non-dimensional variables (denoted by hats);

$$
\hat{x}=\frac{x}{a}, \hat{y}=\frac{y}{b}, \hat{w}=\frac{w}{d}, \hat{u}=\frac{a u}{d^{2}}, \hat{v}=\frac{a v}{d^{2}}, \hat{t}=\frac{t}{T}
$$

Substituting equation (10) into equations (1)-(3) and dropping the hats we get the following equations:

$$
\frac{\partial^{2} u}{\partial x^{2}}+\frac{(1+v)}{2 \alpha} \frac{\partial^{2} v}{\partial x \partial y}+\frac{(1-v)}{2 \alpha^{2}} \frac{\partial^{2} u}{\partial y^{2}}+\frac{\partial w}{\partial x} \frac{\partial^{2} w}{\partial x^{2}}+\frac{(1+v)}{2 \alpha^{2}} \frac{\partial w}{\partial y} \frac{\partial^{2} w}{\partial x \partial y}+\frac{(1-v)}{2 \alpha^{2}} \frac{\partial w}{\partial x} \frac{\partial^{2} w}{\partial y^{2}}=0
$$




$$
\begin{aligned}
& \frac{\partial^{2} v}{\partial y^{2}}+\frac{(1+v) \alpha}{2} \frac{\partial^{2} u}{\partial x \partial y}+\frac{(1-v) \alpha^{2}}{2} \frac{\partial^{2} v}{\partial x^{2}}+\frac{1}{\alpha} \frac{\partial w}{\partial y} \frac{\partial^{2} w}{\partial y^{2}}+\frac{(1+v) \alpha}{2} \frac{\partial w}{\partial x} \frac{\partial^{2} w}{\partial x \partial y}+\frac{(1-v)}{2} \frac{\partial w}{\partial y} \frac{\partial^{2} w}{\partial x^{2}}=0 \\
& \frac{\partial^{4} w}{\partial x^{4}}+\frac{2}{\alpha^{2}} \frac{\partial^{4} w}{\partial x^{2} \partial y^{2}}+\frac{1}{\alpha^{4}} \frac{\partial^{4} w}{\partial y^{4}}+\frac{\partial w}{\partial t^{2}}+\hat{c} \frac{\partial w}{\partial t}=\alpha_{2} \frac{V(t)^{2}}{(1-w)^{2}}+3 \alpha_{0}^{2}\left\{\hat{N}_{x x} \frac{\partial^{2} w}{\partial x^{2}}+\frac{2}{\alpha} \hat{N}_{x y} \frac{\partial^{2} w}{\partial x \partial y}+\frac{1}{\alpha^{2}} \hat{N}_{y y} \frac{\partial^{2} w}{\partial y^{2}}\right\} \\
& +12 \alpha_{1}^{2}\left\{\frac{\partial u}{\partial x}\left(\frac{\partial^{2} w}{\partial x^{2}}+\frac{v}{\alpha^{2}} \frac{\partial^{2} w}{\partial y^{2}}\right)+\frac{1}{\alpha} \frac{\partial v}{\partial y}\left(v \frac{\partial^{2} w}{\partial x^{2}}+\frac{1}{\alpha^{2}} \frac{\partial^{2} w}{\partial y^{2}}\right)+(1-v)\left(\frac{1}{\alpha} \frac{\partial u}{\partial y}+\frac{\partial v}{\partial x}\right) \frac{1}{\alpha} \frac{\partial^{2} w}{\partial x \partial y}\right\} \\
& +12 \alpha_{1}^{2}\left\{\frac{1}{2}\left(\frac{\partial w}{\partial x}\right)^{2}\left(\frac{\partial^{2} w}{\partial x^{2}}+\frac{v}{\alpha^{2}} \frac{\partial^{2} w}{\partial y^{2}}\right)+\frac{1}{2 \alpha^{2}}\left(\frac{\partial w}{\partial y}\right)^{2}\left(v \frac{\partial^{2} w}{\partial x^{2}}+\frac{1}{\alpha^{2}} \frac{\partial^{2} w}{\partial y^{2}}\right)+\frac{(1-v)}{\alpha^{2}}\left(\frac{\partial w}{\partial x} \frac{\partial w}{\partial y} \frac{\partial^{2} w}{\partial x \partial y}\right)\right\}
\end{aligned}
$$

The parameters appearing in equations (11)-(13) are

$$
\alpha=\frac{b}{a}, \quad \alpha_{0}=\frac{a}{h}, \quad \alpha_{1}=\frac{d}{h}, \quad \alpha_{2}=\frac{6\left(1-v^{2}\right)}{E h^{3} d^{3}} \varepsilon a^{4}, \hat{c}=\frac{c a^{4}}{T D} \text { and } \hat{N}_{i j}=\frac{\left(1-v^{2}\right)}{E h} N_{i j}
$$

Where $T$ is the characteristic time expressed as $T=\sqrt{\frac{\rho a^{4}}{D}}$ and $D$ is the flexural rigidity of the plate expressed as $D=\frac{E h^{3}}{12\left(1-v^{2}\right)}$.

The boundary conditions for the non-dimensional equations are

$$
\begin{gathered}
u(0, y, t)=v(0, y, t)=u(1, y, t)=v(1, y, t)=0 \\
u(x, 0, t)=v(x, 0, t)=u(x, 1, t)=v(x, 1, t)=0 \\
w(0, y, t)=w(1, y, t)=0 \\
\frac{\partial w}{\partial x}(0, y, t)=\frac{\partial w}{\partial x}(1, y, t)=0 \\
w(x, 0, t)=w(x, 1, t)=0 \\
\frac{\partial w}{\partial y}(x, 0, t)=\frac{\partial w}{\partial y}(x, 1, t)=0
\end{gathered}
$$

For the purpose of solving for the eigenvalues of the plate, we linearize the non-dimensional governing equations (11)-(13) around the deflected position due the DC electrostatic load. 
Towards this, we express the microplate response as the sum of the static components $w_{s}, u_{s}$ and $v_{s}$, and the dynamic components $w_{d}, u_{d}$, and $v_{d}$

$$
\begin{aligned}
& w=w_{s}+w_{d} \\
& u=u_{s}+u_{d} \\
& v=v_{s}+v_{d}
\end{aligned}
$$

Now, by plugging equations (21)-(23) into equations (11)-(13), cancelling the equilibrium terms and retaining only linear terms in $u_{d}, v_{d}$ and $w_{d}$, we obtain

$$
\begin{aligned}
& \frac{\partial^{2} u_{d}}{\partial x^{2}}+\frac{(1+v)}{2 \alpha} \frac{\partial^{2} v_{d}}{\partial x \partial y}+\frac{(1-v)}{2 \alpha^{2}} \frac{\partial^{2} u_{d}}{\partial y^{2}}+\frac{\partial w_{s}}{\partial x} \frac{\partial^{2} w_{d}}{\partial x^{2}}+\frac{\partial w_{d}}{\partial x} \frac{\partial^{2} w_{s}}{\partial x^{2}}+\frac{(1+v)}{2 \alpha^{2}}\left(\frac{\partial w_{s}}{\partial y} \frac{\partial^{2} w_{d}}{\partial x \partial y}+\frac{\partial w_{d}}{\partial y} \frac{\partial^{2} w_{s}}{\partial x \partial y}\right) \\
& +\frac{(1-v)}{2 \alpha^{2}}\left(\frac{\partial w_{s}}{\partial x} \frac{\partial^{2} w_{d}}{\partial y^{2}}+\frac{\partial w_{d}}{\partial x} \frac{\partial^{2} w_{s}}{\partial y^{2}}\right)=0 \\
& \frac{\partial^{2} v_{d}}{\partial y^{2}}+\frac{(1+v) \alpha}{2} \frac{\partial^{2} u_{d}}{\partial x \partial y}+\frac{(1-v) \alpha^{2}}{2} \frac{\partial^{2} v_{d}}{\partial x^{2}}+\frac{1}{\alpha}\left(\frac{\partial w_{s}}{\partial y} \frac{\partial^{2} w_{d}}{\partial y^{2}}+\frac{\partial w_{d}}{\partial y} \frac{\partial^{2} w_{s}}{\partial y^{2}}\right)+\frac{(1+v) \alpha}{2}\left(\frac{\partial w_{s}}{\partial x} \frac{\partial^{2} w_{d}}{\partial x \partial y}+\frac{\partial w_{d}}{\partial x} \frac{\partial^{2} w_{s}}{\partial x \partial y}\right) \\
& +\frac{(1-v)}{2}\left(\frac{\partial w_{s}}{\partial y} \frac{\partial^{2} w_{d}}{\partial x^{2}}+\frac{\partial w_{d}}{\partial y} \frac{\partial^{2} w_{s}}{\partial x^{2}}\right)=0 \\
& \frac{\partial^{4} w_{d}}{\partial x^{4}}+\frac{2}{\alpha^{2}} \frac{\partial^{4} w_{d}}{\partial x^{2} \partial y^{2}}+\frac{1}{\alpha^{4}} \frac{\partial^{4} w_{d}}{\partial y^{4}}+\frac{\partial w_{d}}{\partial t^{2}}=2 \alpha_{2} \frac{V(t)^{2} w_{d}}{\left(1-w_{s}\right)^{3}}+3 \alpha_{0}^{2}\left\{\hat{N}_{x x} \frac{\partial^{2} w}{\partial x^{2}}+\frac{2}{\alpha} \hat{N}_{x y} \frac{\partial^{2} w}{\partial x \partial y}+\frac{1}{\alpha^{2}} \hat{N}_{y y} \frac{\partial^{2} w}{\partial y^{2}}\right\} \\
& +12 \alpha_{1}^{2}\left\{\begin{array}{l}
\frac{\partial u_{s}}{\partial x}\left(\frac{\partial^{2} w_{d}}{\partial x^{2}}+\frac{v}{\alpha^{2}} \frac{\partial^{2} w_{d}}{\partial y^{2}}\right)+\frac{\partial u_{d}}{\partial x}\left(\frac{\partial^{2} w_{s}}{\partial x^{2}}+\frac{v}{\alpha^{2}} \frac{\partial^{2} w_{s}}{\partial y}\left(v \frac{\partial^{2} w_{d}}{\partial x^{2}}+\frac{1}{\alpha^{2}} \frac{\partial^{2} w_{d}}{\partial y^{2}}\right)+\frac{1}{\alpha} \frac{\partial v_{d}}{\partial y}\left(v \frac{\partial^{2} w_{s}}{\partial x^{2}}+\frac{1}{\alpha^{2}} \frac{\partial^{2} w_{s}}{\partial y^{2}}\right)\right. \\
+(1-v)\left(\frac{1}{\alpha} \frac{\partial u_{s}}{\partial y}+\frac{\partial v_{s}}{\partial x}\right) \frac{1}{\alpha} \frac{\partial^{2} w_{d}}{\partial x \partial y}+(1-v)\left(\frac{1}{\alpha} \frac{\partial u_{d}}{\partial y}+\frac{\partial v_{d}}{\partial x}\right) \frac{1}{\alpha} \frac{\partial^{2} w_{s}}{\partial x \partial y}
\end{array}\right\} \\
& \left(\frac{1}{2}\left(\frac{\partial w_{s}}{\partial x}\right)^{2}\left(\frac{\partial^{2} w_{d}}{\partial x^{2}}+\frac{v}{\alpha^{2}} \frac{\partial^{2} w_{d}}{\partial y^{2}}\right)+\left(\frac{\partial w_{s}}{\partial x}\right)\left(\frac{\partial w_{d}}{\partial x}\right)\left(\frac{\partial^{2} w_{s}}{\partial x^{2}}+\frac{v}{\alpha^{2}} \frac{\partial^{2} w_{s}}{\partial y^{2}}\right)\right. \\
& +12 \alpha_{1}^{2}\left\{\begin{array}{l}
+\frac{1}{2 \alpha^{2}}\left(\frac{\partial w_{s}}{\partial y}\right)^{2}\left(v \frac{\partial^{2} w_{d}}{\partial x^{2}}+\frac{1}{\alpha^{2}} \frac{\partial^{2} w_{d}}{\partial y^{2}}\right)+\frac{1}{2 \alpha^{2}}\left(\frac{\partial w_{s}}{\partial y}\right)\left(\frac{\partial w_{d}}{\partial y}\right)\left(v \frac{\partial^{2} w_{s}}{\partial x^{2}}+\frac{1}{\alpha^{2}} \frac{\partial^{2} w_{s}}{\partial y^{2}}\right) \\
+\frac{(1-v)}{\alpha^{2}}\left(\frac{\partial w_{s}}{\partial x} \frac{\partial w_{s}}{\partial y} \frac{\partial^{2} w_{d}}{\partial x \partial y}\right)+\frac{(1-v)}{\alpha^{2}}\left(\frac{\partial w_{s}}{\partial x} \frac{\partial w_{d}}{\partial y} \frac{\partial^{2} w_{s}}{\partial x \partial y}\right)+\frac{(1-v)}{\alpha^{2}}\left(\frac{\partial w_{d}}{\partial x} \frac{\partial w_{s}}{\partial y} \frac{\partial^{2} w_{s}}{\partial x \partial y}\right)
\end{array}\right\}
\end{aligned}
$$


Equations (24)-(26) are the linearized governing equations of a microplate initially deflected by an electrostatic DC load. These equations are not suitable for analyzing large amplitude vibration of the plates; and hence will be only used to determine the eigenvalues under the action of the electrostatic forces. The forced vibration analysis will be based on the full nonlinear equations, equations (11)-(13).

\section{REDUCED ORDER MODELS}

In this section we discuss the various approaches to develop the reduced order model for the governing equations.

\section{Model I}

In the first approach we develop a semi-reduced order model of the governing equations (11)(13). The governing equation of the out-of-plane motion, equation (13), is reduced into a finitedegree-of-freedom system using the Galerkin method. We use the product of a beam modeshapes along $x$ and $y$ as basis functions in the Galerkin procedure. Hence, we express the out-of-plane deflection $w$ as

$$
w=\sum_{i=1}^{m} q_{i}(t) \phi_{i}^{j, k}(x, y)
$$

where $\phi_{i}^{j, k}(x, y)$ are the basis functions constructed by taking product of the clamped-clamped beam modes; $j$ th mode along $x$ and $k$ th mode along $y$ axis, $j, k=1,3,5 \ldots$, and $q_{i}(t)$ are the unknown time dependent coefficients. We multiply equation (13) by $(1-w)^{2}$ to incorporate the electric force term exactly and simplify dealing with the electrostatic force term. Substituting equation (27) into equation (13), multiplying by $\phi_{i}^{j, k}(x, y)$ and integrating over the domain we 
obtain a discretized form of the out-of-plane equation of motion. The result is a system of nonlinear ordinary differential equations (ODEs) in the time dependent coefficients $q_{i}(t)$, coupled with the in-plane partial differential equations, (11) and (12).

This semi-ROM is solved iteratively using a combination of the Runge Kutta method along with the method of lines. The solution method is outlined in the following:

1. First we put $u=0$ and $v=0$ into the system of discretized ODEs, apply the direct voltage $V_{d c}$ and solve for the out-of-plane deflection $w$.

2. Next plug the deflection $w$ into equations (11) and (12) and solve them for $u$ and $v$.

3. Next the solution for $u$ and $v$ is substituted into the system of the discretized ODEs to solve for the deflection $w$ under an applied voltage $V_{d c}$.

4. Repeat the procedure until a converged solution for $w$ is obtained.

This model can work well for static simulations. However, it is not convenient for dynamic analysis due to its iterative nature.

\section{Model II}

In the second approach we develop a full ROM of the governing equations (11)-(13). The difference between this model and the Model I is that we discretize all three equations of motion using beam modeshapes. The Galerkin procedure for the out-of-plane motion, equation (13), is the same as described in the Model I. To discretize equations (11) and (12) of the inplane motion, we express the in-plane displacements $u$ and $v$ as

$$
u=q_{u}(t) \phi^{2,1}(x, y)
$$




$$
v=q_{v}(t) \phi^{1,2}(x, y)
$$

where $\phi^{2,1}(x, y)$ and $\phi^{1,2}(x, y)$ are the shape-functions constructed by multiplying modeshapes of a clamped-clamped beam; where the first superscript refers to the mode along the $x$ axis and the second superscript refers to the mode along the $y$ axis, $q_{u}(t)$ and $q_{v}(t)$ are the corresponding time dependent coefficients. Substituting equations (28) and (29) into equations (11) and (12), respectively, multiplying by $\phi^{2,1}(x, y)$ and $\phi^{1,2}(x, y)$, respectively, and integrating over the domain we get a set of algebraic equations in the time dependent coefficients $q_{u}(t)$ and $q_{v}(t)$. This set of algebraic equations combined with the system of nonlinear ODEs produced in the Model I constitute the full ROM of the governing equations. Numerical integration of the system of differential algebraic equations (DAEs) can be performed using the Runge Kutta method to obtain a solution for the time dependent coefficients $q_{i}(t), q_{u}(t)$ and $q_{v}(t)$, which are substituted back into equations (27)-(29) to get $w, u$ and $v$.

\section{Model III}

In the third model, we use the modeshapes of the plate calculated using a FEM analysis as the basis functions in the Galerkin procedure. We use the commercial software COMSOL [36] to obtain the modeshapes of the plate. Accordingly, we express the displacement variables $w, u$ and $v$ as

$$
\begin{gathered}
w=\sum_{i=1}^{m} q_{i}(t) \phi_{i}(x, y) \\
u=q_{u}(t) \psi_{u}(x, y) \\
v=q_{v}(t) \psi_{v}(x, y)
\end{gathered}
$$


where $\phi_{i}(x, y)$ are out-of-plane modeshapes of the plate while $\psi_{u}(x, y)$ and $\psi_{v}(x, y)$ are the inplane modeshapes and $q_{i}(t), q_{u}(t)$ and $q_{v}(t)$ are the corresponding unknown time dependent coefficients. Equation (13) is multiplied by $(1-w)^{2}$ to treat the electric force term in its exact form. Substituting equations (30)-(32) into equations (11)-(13), multiplying equation (13) by $\phi_{i}(x, y)$ and equations (11) and (12) by $\psi_{u}(x, y)$ and $\psi_{v}(x, y)$, respectively, and integrating over the domain we get the reduced order model of the governing equations. The reduced order model comprises of a system of differential algebraic equations (DAEs) in the unknown time dependent coefficients $q_{i}(t), q_{u}(t)$ and $q_{v}(t)$, which as before can be solved for $q_{i}(t), q_{u}(t)$ and $q_{v}(t)$ using numerical integration and the Runge Kutta method.

\section{Model IV}

This model is similar to the Model III; the deflection and in-plane displacement variables $w, u$ and $v$ are expressed as in equations (30)-(32). In this model $\phi_{i}(x, y)$ are the same out-of-plane modeshapes of the plate, obtained using FEM (Fig. 8), while $\psi_{u}(x, y)$ and $\psi_{v}(x, y)$ are the shape functions for the in-plane displacements $u$ and $v$ (Fig. 7),. Here, we do not use regular modeshapes for the in-plane displacement. Instead, we make use of the fact the in-plane inertia is negligible, and hence, obtain instead, in-plane shape functions from FEM assuming the plate is deflected by a uniform transverse pressure. Both modeshapes $\phi_{i}(x, y)$ and shape functions $\psi_{u}(x, y)$ and $\psi_{v}(x, y)$ are obtained using FEM Software COMSOL. A mesh convergence test is run to choose a suitable mesh. Results of the mesh convergence test and the modeshapes of the plate along with the in-plane displacement shape functions are given in the 
Appendix. Other than the basis functions, the Galerkin procedure is the same as in the Model III and the resulting ROM consists of DAEs as well.

\section{Model V}

This approach follows the method outlined in [35]. In this approach, the out-of-plane deflection $w$ is expressed as in equation (30). Substituting equation (30) into equations (11) and (12) we obtain the following equations:

$$
\begin{aligned}
& \frac{\partial^{2} u}{\partial x^{2}}+\frac{(1+v)}{2 \alpha} \frac{\partial^{2} v}{\partial x \partial y}+\frac{(1-v)}{2 \alpha^{2}} \frac{\partial^{2} u}{\partial y^{2}}+\sum_{k, l} q_{k} q_{l}\left[\left(\frac{\partial \phi_{k}}{\partial x} \frac{\partial^{2} \phi_{l}}{\partial x^{2}}+\frac{(1+v)}{2 \alpha^{2}} \frac{\partial \phi_{k}}{\partial y} \frac{\partial^{2} \phi_{l}}{\partial x \partial y}\right)+\frac{(1-v)}{2 \alpha^{2}}\left(\frac{\partial \phi_{k}}{\partial x} \frac{\partial^{2} \phi_{l}}{\partial y^{2}}\right)\right]=0 \\
& \frac{\partial^{2} v}{\partial y^{2}}+\frac{(1+v) \alpha}{2} \frac{\partial^{2} u}{\partial x \partial y}+\frac{(1-v) \alpha^{2}}{2} \frac{\partial^{2} v}{\partial x^{2}}+\sum_{k, l} q_{k} q_{l}\left[\left(\frac{1}{\alpha} \frac{\partial \phi_{k}}{\partial y} \frac{\partial^{2} \phi_{l}}{\partial y^{2}}+\frac{(1+v) \alpha}{2} \frac{\partial \phi_{k}}{\partial x} \frac{\partial^{2} \phi_{l}}{\partial x \partial y}\right)+\frac{(1-v)}{2}\left(\frac{\partial \phi_{k}}{\partial y} \frac{\partial^{2} \phi_{l}}{\partial x^{2}}\right)\right]=0
\end{aligned}
$$

Equations (33) and (34) are nonhomogeneous coupled linear PDEs in $u$ and $v$. Since these equations are linear in $u$ and $v$, the principle of superposition can be used to determine the solution for $u$ and $v$ in the form [37]

$$
\begin{aligned}
& u=\sum_{k, l} q_{k} q_{l} u^{k l} \\
& v=\sum_{k, l} q_{k} q_{l} v^{k l}
\end{aligned}
$$

where the superscript $k l$ denotes the in-plane displacements $u^{k l}$ and $v^{k l}$ caused by the loads $f_{x}^{k l}$ and $f_{y}^{k l}$ defined as

$$
\begin{aligned}
& f_{x}^{k l}=\left(\frac{\partial \phi_{k}}{\partial x} \frac{\partial^{2} \phi_{l}}{\partial x^{2}}+\frac{(1+v)}{2 \alpha^{2}} \frac{\partial \phi_{k}}{\partial y} \frac{\partial^{2} \phi_{l}}{\partial x \partial y}\right)+\frac{(1-v)}{2 \alpha^{2}}\left(\frac{\partial \phi_{k}}{\partial x} \frac{\partial^{2} \phi_{l}}{\partial y^{2}}\right) \\
& f_{y}^{k l}=\left(\frac{1}{\alpha} \frac{\partial \phi_{k}}{\partial y} \frac{\partial^{2} \phi_{l}}{\partial y^{2}}+\frac{(1+v) \alpha}{2} \frac{\partial \phi_{k}}{\partial x} \frac{\partial^{2} \phi_{l}}{\partial x \partial y}\right)+\frac{(1-v)}{2}\left(\frac{\partial \phi_{k}}{\partial y} \frac{\partial^{2} \phi_{l}}{\partial x^{2}}\right)
\end{aligned}
$$


Equation (13) is multiplied by $(1-w)^{2}$ to treat the electric force term exactly with no approximation. Now by substituting equations (30), (35), and (36) into equation (13), multiplying by $\phi_{i}(x, y)$ and integrating over the domain we get the reduced order model for the governing equations. The reduced order model consists of a system of nonlinear coupled ODEs in $q_{i}(t)$. This system of ODEs is solved for $q_{i}(t)$ numerically using the Runge Kutta method and substituted back into equation (30), (35) and (36) to obtain $w, u$ and $v$, respectively.

\section{STATIC ANALYSIS}

To calculate the static deflection of the microplate under a DC load, $V(\mathrm{t})$ is replaced by $V_{d c}$. We then drop the time derivatives in all the models and the time dependent unknown coefficient $q_{i}(t)$ in the semi-ROM, and $q_{i}(t), q_{u}(t)$ and $q_{v}(t)$ in all other models are written as constant coefficients $q_{i}, q_{u}$ and $q_{v}$. This results in a system of nonlinear algebraic equations, which is numerically solved for $q_{i}$ in case of the semi-ROM and $q_{i}, q_{u}$ and $q_{v}$ in all other models. Then equation (27) or equation (30) is used to find the transverse deflection.

First we study the convergence of the static results with the number of transverse modes, $\phi_{i}(x, y)$ retained in the reduced order model when $\alpha=1$ and $\alpha_{1}=1$. Figure 2 shows the stable solutions, the non-dimensional deflection $W_{\max }$ at the center of the plate against various values of $\alpha_{2} V_{d c}{ }^{2}$. These convergence static results are based on non-dimensional parameters and hence are not specific to any plate of a particular geometry or material. A summary of the convergence results for all the approaches is given in Table 1. Figure 2 indicates a classical behavior of electrostatically actuated microstructures, where the deflection slopes approaches 
infinity at the pull-in voltage, where the structure collapses to the substrate [6]. As clear from the figure and the table, the Model I and II requires in total six transversal modeshapes for convergence, while the Models III-V requires four.

\section{MODEL VALIDATION}

For validation purpose, the static response calculated by the reduced order models is compared with the results obtained from a FE model implemented in COMSOL [36]. The electromechanics module of COMSOL was used to model the electrically actuated microplate. A mapped mesh with fifty divisions along each edge was used. The geometric and material parameters used are; $a=b=300 \mu \mathrm{m}, h=2 \mu \mathrm{m}, d=2 \mu \mathrm{m}, E=153 \mathrm{GPa}$ and $v=0.23$. Since this is a geometrically nonlinear problem, geometric nonlinearity is activated before performing the analysis. Figure 3 shows the maximum deflection $W_{\max }$ at the center of the microplate against $V_{d c}$ up to the pull-in instability. The figure shows excellent agreement between the results calculated by the ROMs and the FE model except the Model III. The Model III predicts the pull-in of the microplate at a lower voltage. This means that it does not account for the cubic nonlinearity accurately and only captures the effect of midplane stretching partially. In other words, we can remark that the shape functions used to represent the in-plane displacements are not accurate enough to capture the full effect of midplane stretching. All the other models accurately predict the static response of the microplate. Although accurate, the Model I and II requires more computational effort as compared with the Model IV and V. Nevertheless they use easy to handle analytical functions as basis function in the discretization procedure. The Model V calculates exact solution for the in-plane displacement $u$ and $v$, hence requires more computational effort for integration over plate domain as compared with the Model IV, which approximates the solution 
through equation (31) and (32). Reduced order models are known to be time efficient as compared with the full order finite element models. To highlight the advantage of using reduced order models over finite element model, we compare the time taken to solve the system when a DC voltage, $V_{d c}=60 \mathrm{~V}$ was applied. Results for this comparison are given in Table 2. One can note that time taken by full order FE model is much higher than the reduced order model because it solves thousands of equations, one for each degrees of freedom [4].

\section{DYNAMIC RESPONSE}

First we present the Eigen frequency analysis of the microplate when deflected under the DC voltage $V_{d c}$. The approaches presented in the Models II-V are used in conjunction with equations (24)-(26) to calculate the linear natural frequency of the microplate at different levels of initial deflection $w_{s}$ under the electrostatic voltage $V_{d c}$, until the pull-in. The results are compared in Fig. 4 with the natural frequencies calculated by the FE model implemented in COMSOL. The Model I is not included in the analysis due to its iterative nature. Results calculated by the ROMs show good agreement with the ones calculated by FEM model except for the Model III, which shows significant deviation. This result agrees with the static results

(Fig. 3) and confirms the remark that the Model III does not account well for the cubic nonlinearity due to midplane stretching and that the shape functions used to represent the inplane displacements are not accurate enough to capture the full effect of midplane stretching.

The Models II, IV and V accurately predict the non-dimensional natural frequencies of the microplate. Although accurate, the Model II entails more computational effort as compared with the Model IV and V, since it requires more modes for convergence (Table 1). However it 
employs easy to use analytical functions as basis function in the Galerkin discretization. The Model IV approximates the solution for the in-plane displacements $u$ and $v$ through equation (31) and (32) while the Model V calculates exact solution, hence requires more computational effort. Nonetheless the Model V is easily adaptable to the microplates with other boundary conditions e.g. clamped-free-clamped-free (CFCF). Moreover one can note that the natural frequency decreases with the increase of the applied DC voltage and drops rapidly near the pull-in instability, finally falling to zero at the pull-in voltage. This is due to the softening nature of the electrostatic force. The decrease in the natural frequency of the plate with the increase in the DC load means that its elastic restoring force is weakening, and hence reaches eventually to zero when the natural frequency vanishes.

Next we present the dynamic response of the microplate calculated by the Models II-V, when actuated with small and large electrostatic forces. We use the same number of modes as determined by static convergence test summarized in Table 1. Frequency response curves are generated by actuating the microplate by a harmonic load of amplitude $V_{a c}$ superimposed to $V_{d c}$ load, i.e., $V(\mathrm{t})=V_{d c}+V_{a c} \operatorname{Sin}(\Omega \mathrm{t})$. The Runge Kutta method is used to perform long time numerical integration to solve the system of governing equations to obtain a stable steady solution. A stable steady solution is captured such that transient response is not contributing to the solution. We fix the non-dimensional parameters as $\alpha=1, \alpha_{1}=1, \alpha_{2}=1$ and assume that the in-plane external forces are zero i.e. $N_{x x}=N_{x y}=N_{y y}=0$. To get a linear response, we actuate the microplate at a small load of $V_{d c}=1 \mathrm{~V}$ and a harmonic load of $V_{a c}=0.01 \mathrm{~V}$ (Fig. 5a). For the large amplitude vibration response we use $V_{a c}=1 \mathrm{~V}$ while $V_{d c}$ is kept the same. Figure 5 shows a comparison of the dynamic responses calculated by the Models II-V using frequency response curves for the small and large amplitude vibrations of the microplate. All the the models show good agreement when actuated at small electrostatic loads (Fig. 5a). All the 
models show the linear response very close to each other, the model II shows a very slight difference of less than $1 \%$ from other models. This small difference from other models appears due to the different kind of shape functions used in this model, which seem not of good accuracy.

The Model III shows a significant deviation from the other models, which show an excellent agreement when actuated at large electrostatic loads (Fig. 5b). This agrees with the static deflection (Fig. 3) and natural frequency results (Fig. 4) and ratifies the remark that the Model III does not account accurately for the cubic nonlinearity. For small actuating forces (Fig. 5a), all the models show a resonance near the fundamental natural frequency of the plate. The geometric nonlinearity is not activated, and in this regime, the linear plate theory can be used. When large electrostatic loads are used to actuate the large amplitude vibrations of the plate, the geometric nonlinearity due to midplane stretching is activated. The plate shows strong hardening behavior due to the geometric nonlinearity, where the nonlinear resonances appear at higher frequencies. All the models capture the nonlinear hardening responses of the plate accurately except Model III, which fails to capture the nonlinearity accurately. In this regime, the linear plate theory fails and only a model that accounts for the geometric nonlinearity can capture the plate response accurately.

Although it sounds appropriate to use any of the Models II, IV and V for investigation of large amplitude vibrations of microplates, we believe that the Model $\mathrm{V}$ is the most accurate one since it calculates the exact solution for midplane stretching while for all the other models we approximate the in-plane displacements using some kind of shape functions. Moreover it can be used with confidence for microplates with other boundary conditions for example a clamped-free-clamped-free (CFCF) microplate. For such a case the other models may not be used with the same shape functions. So the Model V is more rigorous and versatile and can be used for a microplate with any kind of boundary conditions. 


\section{Summary and Conclusions}

In this article we investigated different approaches to develop a reduced order model for the nonlinear von-Karman microplates actuated electrically to study the static and dynamic behavior. It is worth to mention here that while most of the studied models of this article have been used, especially in linear applications; their application to the MEMS plate under nonlinear electrostatic forces has not been investigated before. We have attempted in this article to shed light on these methods and their applicability to the micro scale while comparing their performance from different points of view. We used different types of basis functions in conjunction with the Galerkin method to discretize the governing equations.

First the convergence of static response was investigated with the number of modes used in the ROM for the different approaches. Then for validation purpose we compared the static response calculated by the models under investigation with the results calculated by FE model implemented in COMSOL. The comparison has indicated that all the models show an excellent agreement with the FE model results except Model III, which was shown to represent poorly the cubic nonlinearity due to midplane stretching due to the used shape functions.

Eigen frequency analysis was performed using the approaches presented in the Models II-V, in conjunction with linearized equations for initially deflected microplate under DC load $V_{d c}$. Eigen frequency results calculated by the current models are compared with the FE results, which show an excellent agreement except the Model III. This agrees with the static results (Fig. 3) and confirms the remark that the Model III does not account well for the cubic nonlinearity and 
that the shape functions used to represent the in-plane displacements are not accurate enough to capture the full effect of midplane stretching.

Next we compared the dynamic response through frequency response curves at a small and large harmonic exciting force for the Models II-V, since the Model I is not suitable for dynamic studies due to its iterative nature it's excluded from the dynamic analysis. We noted that for linear vibrations analysis all the models show an excellent agreement except the Model II, which show a slight difference from the other models. For the large nonlinear vibrations, the Model III deviate from the other models, which was also the case in the static and Eigen frequency analysis and ratifies the remark that the Model III does not account accurately for the cubic nonlinearity. The Model II, IV and V show very good agreement at large amplitude vibrations.

As a conclusion, although it sounds appropriate to use any of them for investigation of large amplitude vibrations of microplates, we believe that the Model V is the most accurate one since it calculates the exact solution for midplane stretching while for all the other models we approximate the in-plane displacements using some kind of shape functions. Moreover it can be used with confidence for microplates with other boundary conditions for example a clamped-free-clamped-free (CFCF) microplate. For such a case the other models may not be used with the same shape functions. So we conclude that the Model V is more rigorous and versatile and can be used for a microplate with any kind of boundary condition. 


\section{APPENDIX: MODESHAPES AND IN-PLANE SHAPE FUNCTIONS}

The COMSOL Multiphysics FEM software [36] has been used for the extraction of modeshapes and the in-plane displacement shape functions. Towards this, we first performed a mesh convergence test. The FEM model consists of a square plate of side length $100 \mu \mathrm{m}$ and thickness of $2 \mu \mathrm{m}$. Figure 6 shows the convergence of the first natural frequency with increasing the number of elements. We conclude from the figure that using a mapped mesh with 100 elements along each side of the square microplate is a reasonable compromise between accuracy and computational effort, since further increase of accuracy comes at much higher computational cost.

In Fig. 7 are shown the in-plane shape functions $\psi_{u}$ and $\psi_{v}$. These shape functions are extracted using the FEM software COMSOL [36]. A square microplate is deflected by applying a uniform transverse pressure. Then the in-plane displacements $u(x, y)$ and $v(x, y)$ are extracted when the deflection at the center of the plate is nearly equal to half the thickness of the microplate. We normalize these displacements to use as shape functions $\psi_{u}$ and $\psi_{v}$ to be used in the Model IV.

Next the eigenvalue problem of the linear undamped square microplate was solved. The first six non-dimensional frequencies, $\lambda=\omega a^{2} \sqrt{\frac{\rho}{D}}$, of the symmetric-symmetric modeshapes are given in Table 3 and corresponding modeshapes are shown in Fig. 8. 


\section{REFERENCES}

1. Vogl, G.W. and A.H. Nayfeh. A reduced-order model for electrically actuated clamped circular plates. in ASME 2003 International Design Engineering Technical Conferences and Computers and Information in Engineering Conference. 2003. American Society of Mechanical Engineers.

2. Younis, M.I., E.M. Abdel-Rahman, and A. Nayfeh, A reduced-order model for electrically actuated microbeam-based MEMS. Microelectromechanical Systems, Journal of, 2003. 12(5): p. 672-680.

3. Zhao, X., E.M. Abdel-Rahman, and A.H. Nayfeh, A reduced-order model for electrically actuated microplates. Journal of Micromechanics and Microengineering, 2004. 14(7): p. 900-906.

4. Nayfeh, A.H., M.I. Younis, and E.M. Abdel-Rahman, Reduced-order models for MEMS applications. Nonlinear Dynamics, 2005. 41(1-3): p. 211-236.

5. Batra, R.C., M. Porfiri, and D. Spinello, Reduced-order models for microelectromechanical rectangular and circular plates incorporating the Casimir force. International Journal of Solids and Structures, 2008. 45(11-12): p. 3558-3583.

6. May, S.F. and R.C. Smith. Reduced-order model design for nonlinear smart system models. in SPIE Smart Structures and Materials+ Nondestructive Evaluation and Health Monitoring. 2009. International Society for Optics and Photonics.

7. Younis, M.I., MEMS Linear and Nonlinear Statics and Dynamics: Mems Linear and Nonlinear Statics and Dynamics. Vol. 20. 2011: Springer.

8. Machauf, A., Y. Nemirovsky, and U. Dinnar, A membrane micropump electrostatically actuated across the working fluid. Journal of Micromechanics and Microengineering, 2005. 15(12): p. 2309-2316.

9. Chao, P.C., C.-W. Chiu, and C. Tsai, A novel method to predict the pull-in voltage in a closed form for micro-plates actuated by a distributed electrostatic force. Journal of Micromechanics and Microengineering, 2006. 16(5): p. 986-998.

10. Nayfeh, A.H. and M.I. Younis, A new approach to the modeling and simulation of flexible microstructures under the effect of squeeze-film damping. Journal of Micromechanics and Microengineering, 2004. 14(2): p. 170-181.

11. Bertarelli, E., R. Ardito, R. Ardito, A. Corigliano, and R. Contro, A plate model for the evaluation of pull-in instability occurrence in electrostatic micropump diaphragms. International Journal of Applied Mechanics, 2011. 3(01): p. 1-19.

12. Ahmad, B. and R. Pratap, Elasto-electrostatic analysis of circular microplates used in capacitive micromachined ultrasonic transducers. Sensors Journal, IEEE, 2010. 10(11): p. 1767-1773.

13. Porfiri, M., Vibrations of parallel arrays of electrostatically actuated microplates. Journal of Sound and Vibration, 2008. 315(4): p. 1071-1085.

14. Srinivas, D., Electromechanical Dynamics of simply-supported micro-plates. International Journal of Computational Engineering Research, 2012. 2(5): p. 2012.

15. Ng, T.Y., T.Y. Jiang, H. Li, K.Y. Lam, and J.N. Reddy, A coupled field study on the non-linear dynamic characteristics of an electrostatic micropump. Journal of Sound and Vibration, 2004. 273(4-5): p. 989-1006.

16. Younis, M.I. and A.H. Nayfeh, Simulation of squeeze-film damping of microplates actuated by large electrostatic load. Journal of Computational and Nonlinear Dynamics, 2007. 2(3): p. 232241.

17. Faris, W.F., Nonlinear dynamics of annular and circular plates under thermal and electrical loadings (PhD Dissertation). https://vtechworks.lib.vt.edu/handle/10919/11100, 2003.

18. Asghari, M., Geometrically nonlinear micro-plate formulation based on the modified couple stress theory. International Journal of Engineering Science, 2012. 51: p. 292-309. 
19. Gholipour, A., H. Farokhi, and M.H. Ghayesh, In-plane and out-of-plane nonlinear size-dependent dynamics of microplates. Nonlinear Dynamics, 2014. 79(3): p. 1771-1785.

20. Zand, M.M. and M. Ahmadian, Characterization of coupled-domain multi-layer microplates in pull-in phenomenon, vibrations and dynamics. International Journal of Mechanical Sciences, 2007. 49(11): p. 1226-1237.

21. Pursula, A., P. Råback, S. Lähteenmäki, and J. Lahdenperä, Coupled FEM simulations of accelerometers including nonlinear gas damping with comparison to measurements. Journal of Micromechanics and Microengineering, 2006. 16(11): p. 2345-2354.

22. Telukunta, S. and S. Mukherjee, Fully Lagrangian modeling of MEMS with thin plates. IEEE/ASME Journal of Microelectromechanical Systems, 2006. 15(4): p. 795-810.

23. Jia, X.L., J. Yang, and S. Kitipornchai, Pull-in instability of geometrically nonlinear micro-switches under electrostatic and Casimir forces. Acta mechanica, 2011. 218(1-2): p. 161-174.

24. Wang, B., S. Zhou, J. Zhao, and X. Chen, Pull-in instability analysis of electrostatically actuated microplate with rectangular shape. International Journal of Precision Engineering and Manufacturing, 2011. 12(6): p. 1085-1094.

25. Mohammadi, V., R. Ansari, M.F. Shojaei, R. Gholami, and S. Sahmani, Size-dependent dynamic pull-in instability of hydrostatically and electrostatically actuated circular microplates. Nonlinear Dynamics, 2013. 73(3): p. 1515-1526.

26. Mukherjee, S., Z. Bao, M. Roman, and N. Aubry, Nonlinear mechanics of MEMS plates with a total Lagrangian approach. Computers \& Structures, 2005. 83(10): p. 758-768.

27. Faris, W.F., E.M. Abdel-Rahman, and A.H. Nayfeh. Mechanical behavior of an electrostatically actuated micropump. in $43 \mathrm{rd}$ AIAA/ASME/ASCE/AHS/ASC Structures, Structural Dynamics, and Materials Conference, Denver, CO. 2002-1303.

28. Zand, M.M. and M. Ahmadian, Vibrational analysis of electrostatically actuated microstructures considering nonlinear effects. Communications in Nonlinear Science and Numerical Simulation, 2009. 14(4): p. 1664-1678.

29. $\mathrm{Fu}, \mathrm{Y}$. and J. Zhang, Active control of the nonlinear static and dynamic responses for piezoelectric viscoelastic microplates. Smart Materials and Structures, 2009. 18(9): p. 095037.

30. Karimzade, A., H. Moeenfard, and M.T. Ahmadian. Nonlinear Analysis of Pull-In Voltage for a Fully Clamped Microplate With Movable Base. in ASME 2012 International Mechanical Engineering Congress and Exposition. 2012. American Society of Mechanical Engineers.

31. Farokhi, H. and M.H. Ghayesh, Nonlinear dynamical behaviour of geometrically imperfect microplates based on modified couple stress theory. International Journal of Mechanical Sciences, 2015. 90: p. 133-144.

32. Ghayesh, M.H. and H. Farokhi, Nonlinear dynamics of microplates. International Journal of Engineering Science, 2015. 86: p. 60-73.

33. Rahaeifard, M., M. Ahmadian, and K. Firoozbakhsh, Vibration analysis of electrostatically actuated nonlinear microbridges based on the modified couple stress theory. Applied Mathematical Modelling, 2015.

34. Nayfeh, A.H. and D.T. Mook, Nonlinear oscillations. 2008: John Wiley \& Sons.

35. Nayfeh, A.H. and P.F. Pai, Linear and nonlinear structural mechanics. 2008: John Wiley \& Sons.

36. COMSOL Multiphysics, (http://www.comsol.com).

37. Lobitz, D., A. Nayfeh, and D. Mook, Non-linear analysis of vibrations of irregular plates. Journal of Sound and Vibration, 1977. 50(2): p. 203-217. 


\section{Table Caption List}

Table 1 Summary of convergence studies, number of transverse modes required for convergence and the value of the non-dimensional parameter $\alpha_{2} V_{d c}{ }^{2}$ at the pull-in instability.

Table 2 A comparison of the time taken by FE model implemented in COMSOL and the reduced order models to solve the system under an electrostatic DC voltage.

Table 3 Frequency parameter $\lambda=\omega a^{2} \sqrt{\frac{\rho}{D}}$ for first six symmetric-symmetric modeshapes of a square microplate calculated using the FEM software COMSOL. 


\section{Figure Captions List}

Fig. 1 A schematic diagram of an electrically actuated fully clamped microplate.

Fig. 2 Convergence of the static results with the number of transversal modeshapes retained in the reduced order models. Variation of the maximum non-dimensional deflection $W_{\max }$ at the center of the microplate with the electrostatic voltage parameter $\alpha_{2} V_{d c}^{2}$ when $\alpha=1$, and $\alpha_{1}=1$ : (a) Model I, (b) Model II, (c ) Model III, (d) Model IV, (e) Model V.

Fig. 3 A comparison of the maximum deflection $W_{\max }(a / 2, b / 2)$ calculated by the reduced order models with the results obtained from FE model implemented in COMSOL for various values of $V_{d c}$, until the pull-in instability: (a) Model I, (b) Model II, (c ) Model III, (d) Model IV, (e) Model V.

Fig. 4 The non-dimensional fundamental natural frequency $\left(\lambda=\omega a^{2} \sqrt{\frac{\rho}{D}}\right)$ of a square microplate for different levels of $V_{d c}$ until pull-in (stars).

Comparison with the results computed by the FE model implemented in COMSOL (diamonds): (a) Model II, (b) Model III, (c ) Model IV, (d) Model V.

Fig. 5 Frequency response curves near non-dimensional fundamental natural frequency, maximum non-dimensional deflection $W_{\max }(0.5,0.5)$ of the microplate against actuating frequency $\Omega$. The responses are captured 
at $V_{d c}=1 \mathrm{~V}$, and (a) $V_{a c}=0.01 \mathrm{~V}$, (b) $V_{a c}=1 \mathrm{~V}$, when $\alpha=1, \alpha_{1}=1, \alpha_{2}=1$, and a quality factor $Q=1000$.

Fig. 6 Mesh convergence study; convergence of the first natural frequency with increasing the number of elements.

Fig. 7 In-plane displacement shape functions for the MODEL IV, (a) $\psi_{u}(x, y)$, (b) $\psi_{v}(x, y)$.

Fig. 8 The first six symmetric-symmetric transversal modeshapes of a square microplate. 

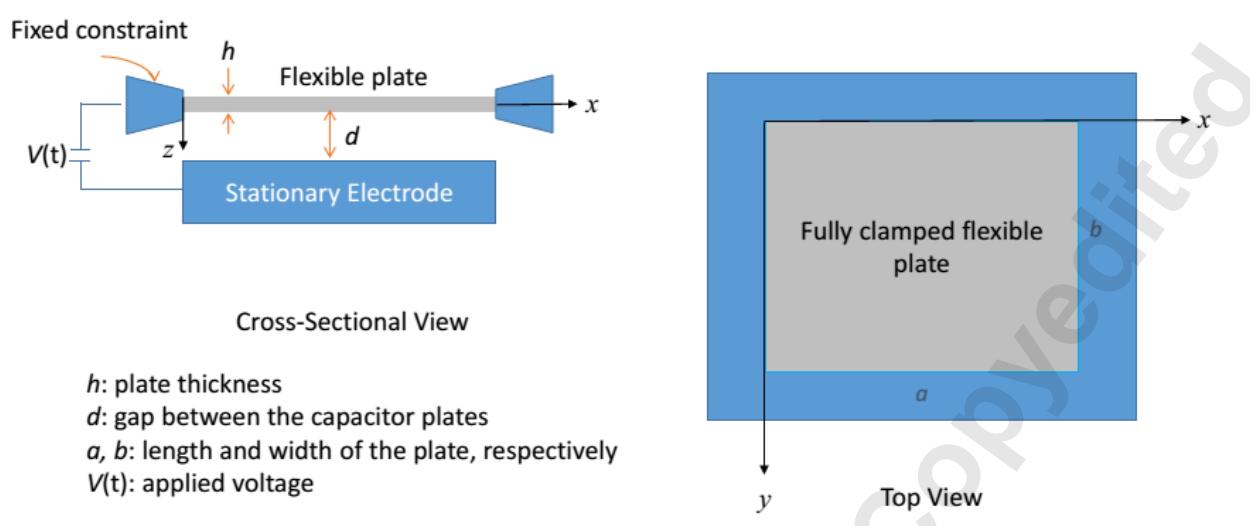

Fig. 1 A schematic diagram of an electrically actuated fully clamped microplate. 


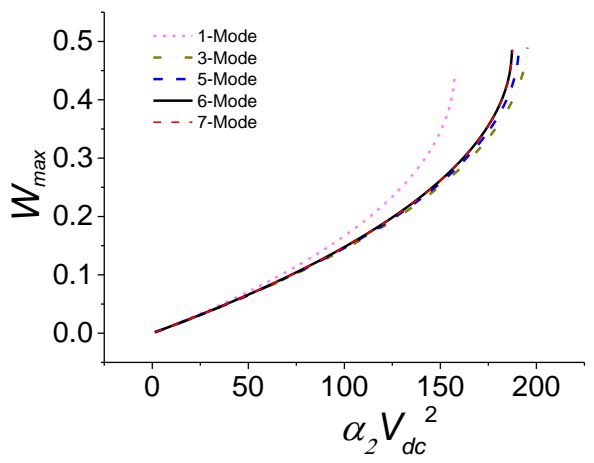

(a)

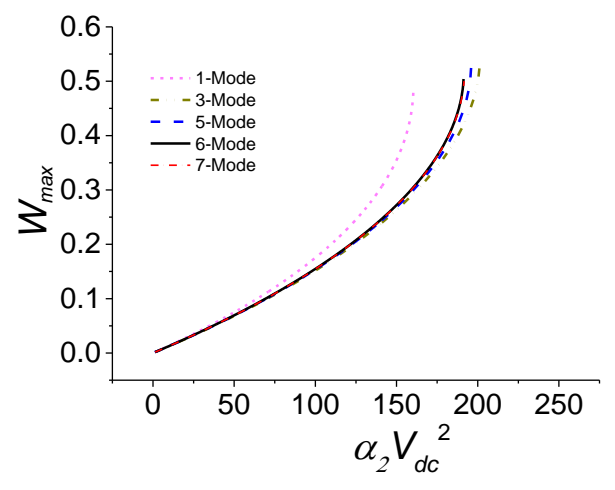

(b)

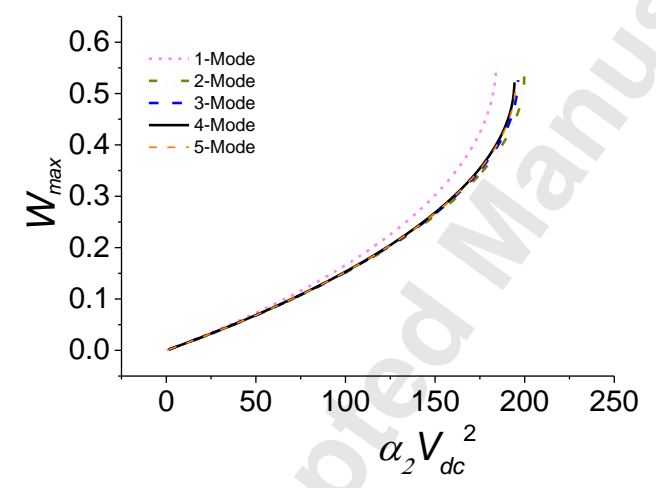

(d)

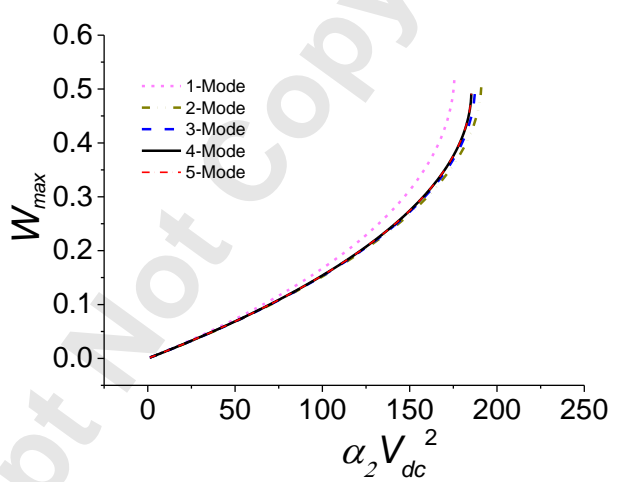

(c)

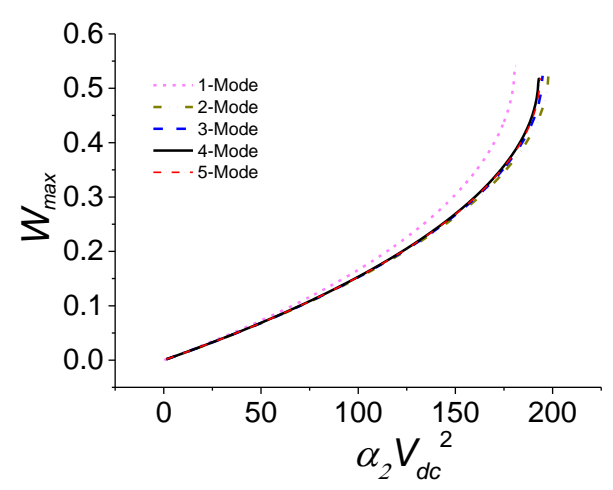

(e)

Fig. 2 Convergence of the static results with the number of transversal modeshapes retained in the reduced order models. Variation of the maximum non-dimensional deflection $W_{\max }$ at the center of the microplate with the electrostatic voltage parameter $\alpha_{2} V_{d c}{ }^{2}$ when $\alpha=1$, and $\alpha_{1}=1$ : (a) Model I, (b) Model II, (c) Model III, (d) Model IV, (e) Model V. 


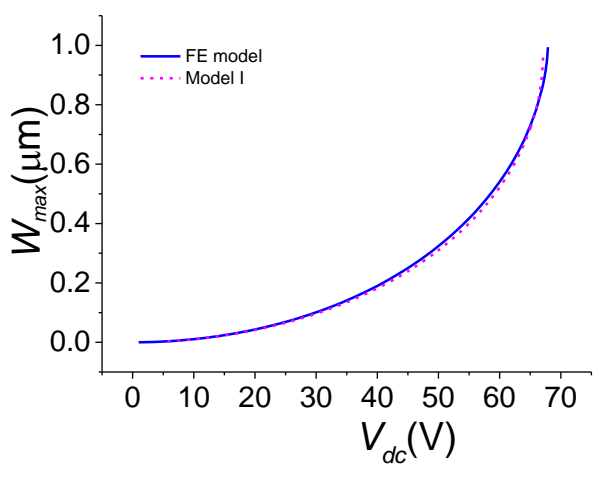

(a)

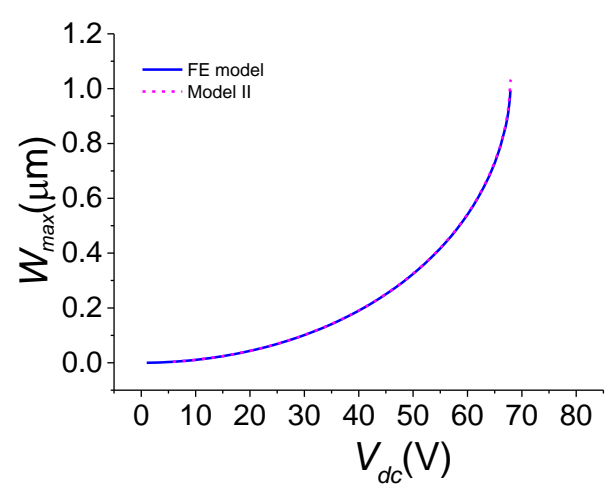

(b)

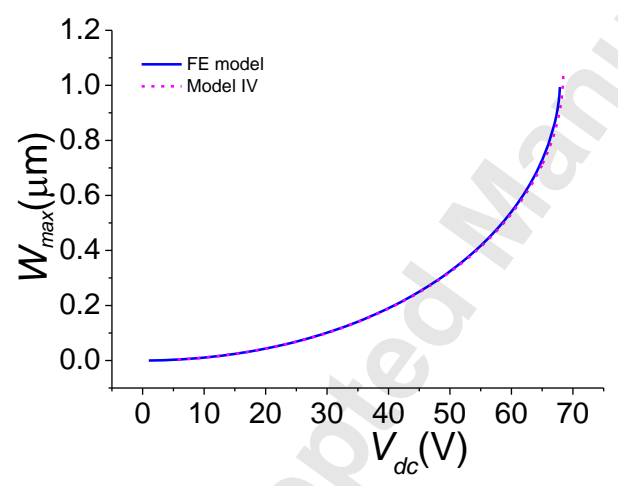

(d)

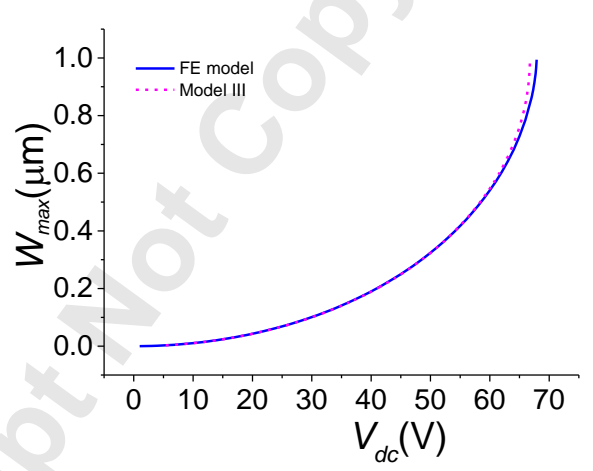

(c)

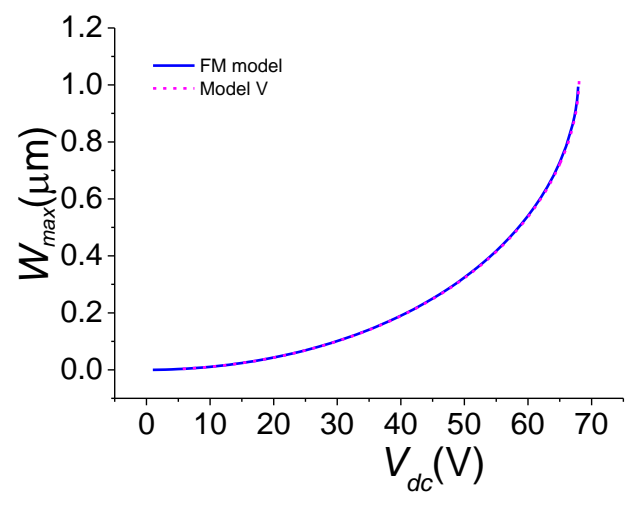

(e)

Fig. 3 A comparison of the maximum deflection $W_{\max }(a / 2, b / 2)$ calculated by the reduced order models with the results obtained from FE model implemented in COMSOL for various values of $V_{d c}$, until the pull-in instability: (a) Model I, (b) Model II, (c ) Model III, (d) Model IV, (e) Model V. 


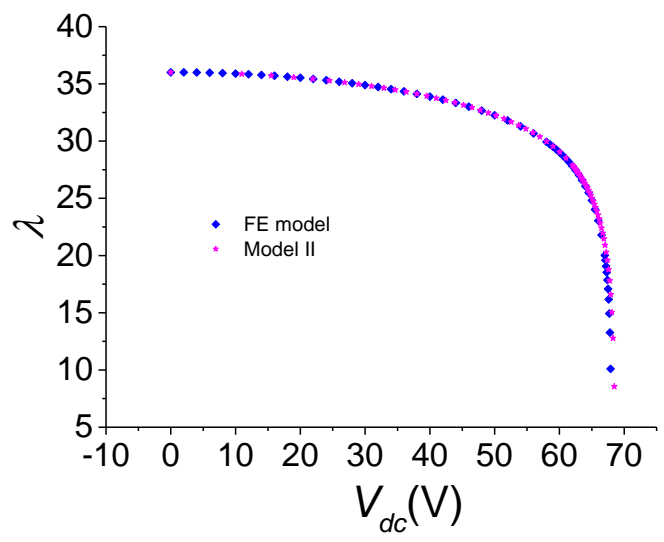

(a)

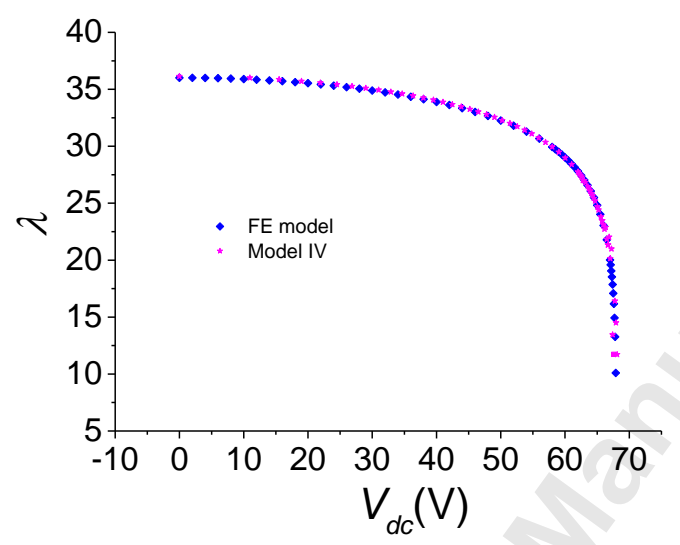

(c)

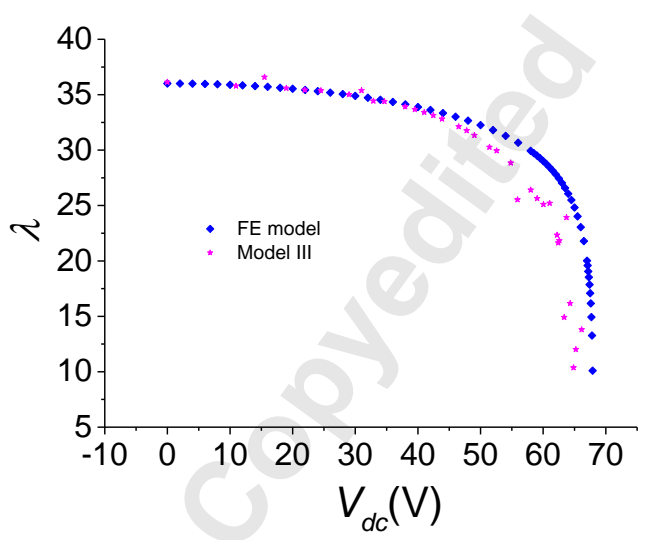

(b)

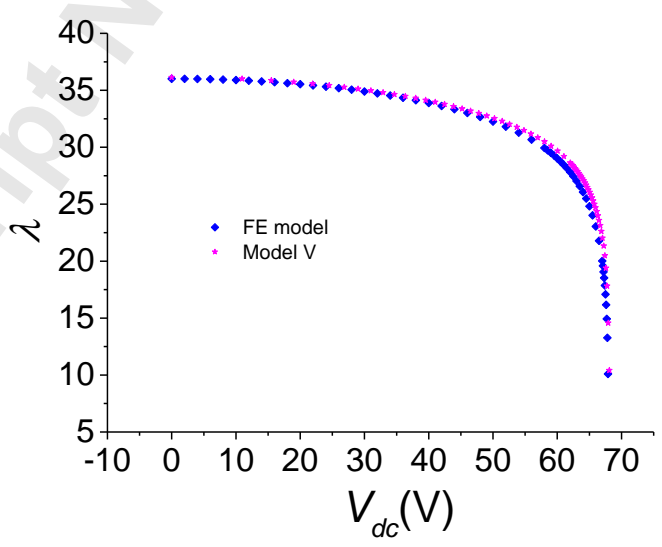

(d)

Fig. 4 The non-dimensional fundamental natural frequency $\left(\lambda=\omega a^{2} \sqrt{\frac{\rho}{D}}\right)$ of a square microplate for different levels of $V_{d c}$ until pull-in (stars). Comparison with the results computed by the FE model implemented in COMSOL (diamonds): (a) Model II, (b) Model III, (c ) Model IV, (d) Model V. 


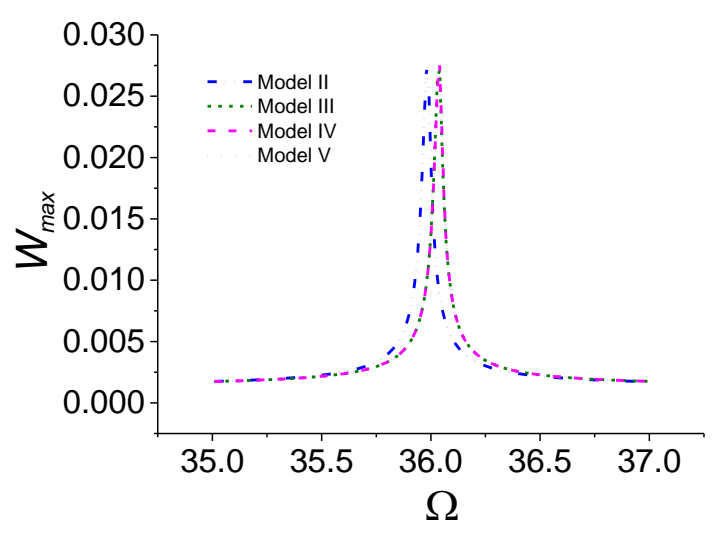

(a)

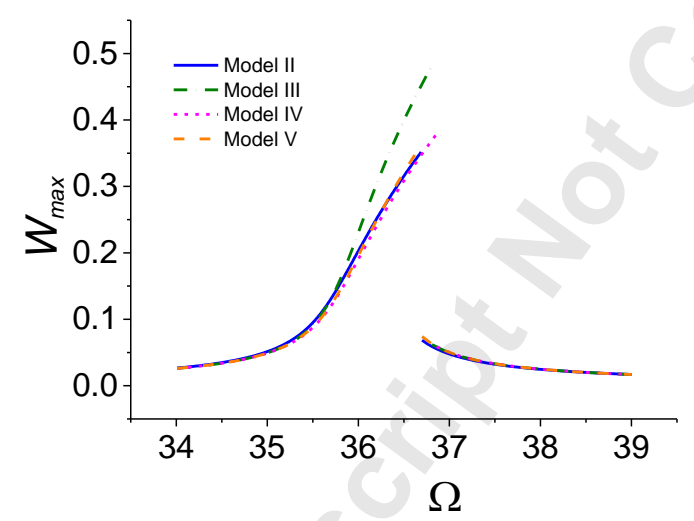

(b)

Fig. 5 Frequency response curves near non-dimensional fundamental natural frequency, maximum non-dimensional deflection $W_{\max }(0.5,0.5)$ of the microplate against actuating frequency $\Omega$. The responses are captured at $V_{d c}=1 \mathrm{~V}$, and (a) $V_{a c}=0.01 \mathrm{~V}$, (b) $V_{a c}=1 \mathrm{~V}$, when $\alpha=1, \alpha_{1}=1, \alpha_{2}=1$, and a quality factor $Q=1000$. 


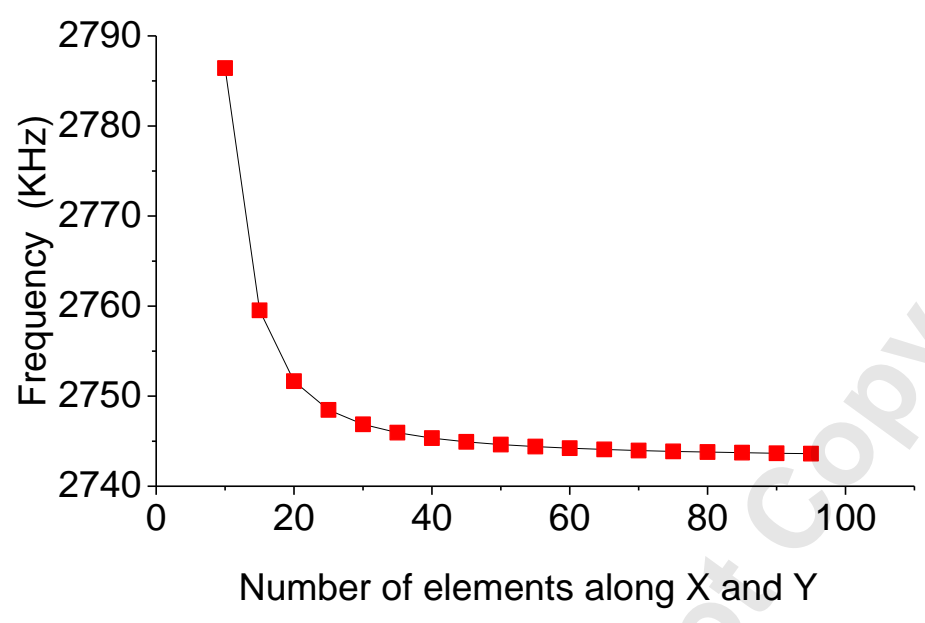

Fig. 6 Mesh convergence study; convergence of the first natural frequency with increasing the number of elements. 


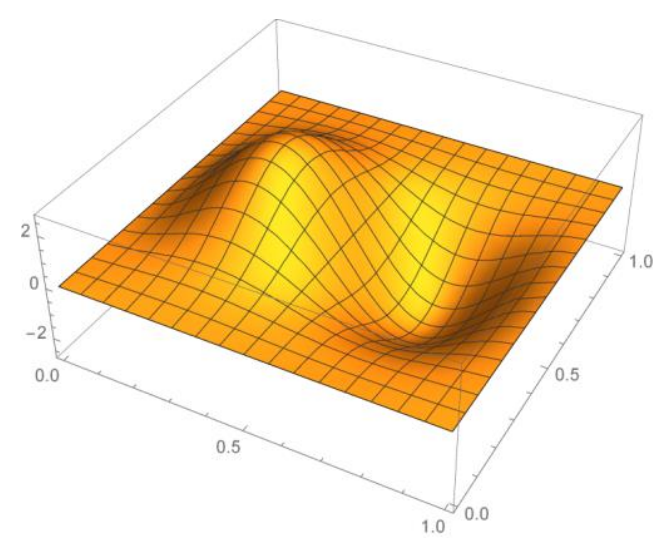

(a)

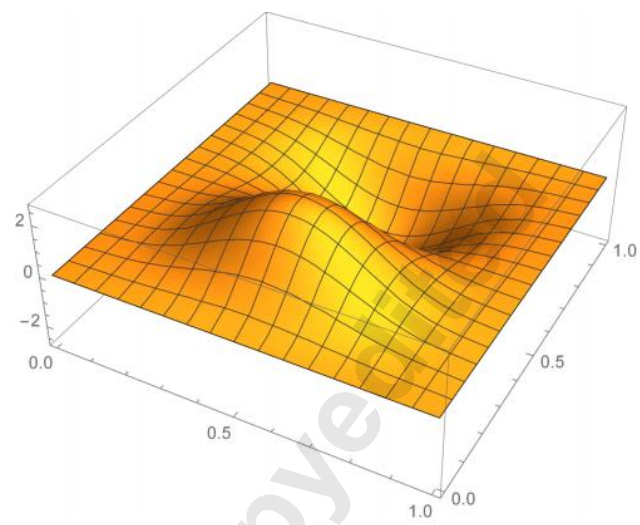

(b)

Fig. 7 In-plane displacement shape functions for the MODEL IV, (a) $\psi_{u}(x, y)$, (b) $\psi_{v}(x, y)$. 


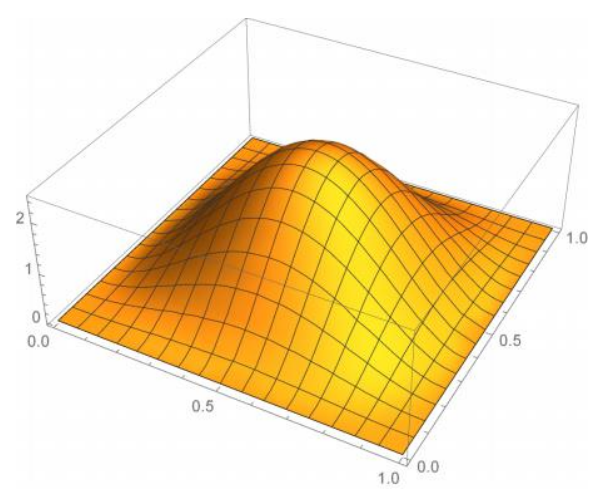

(a)

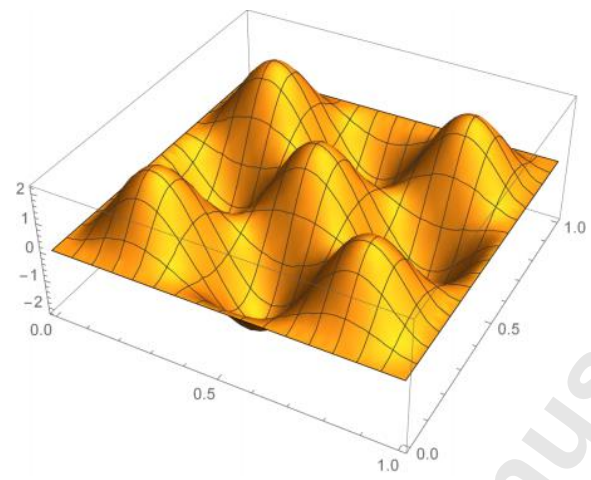

(c)

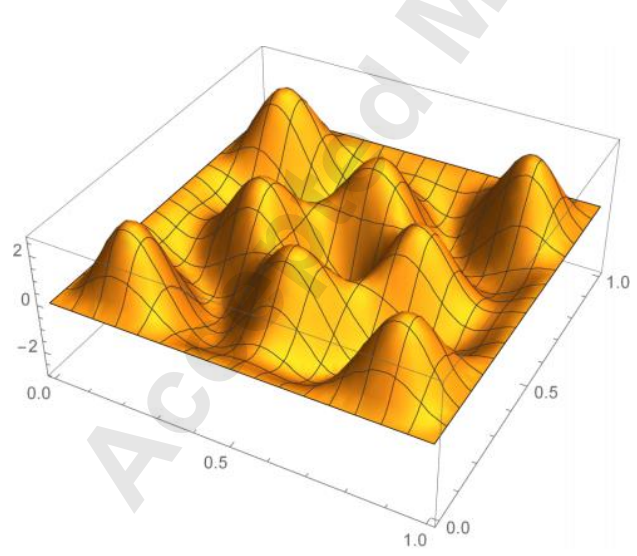

(e)

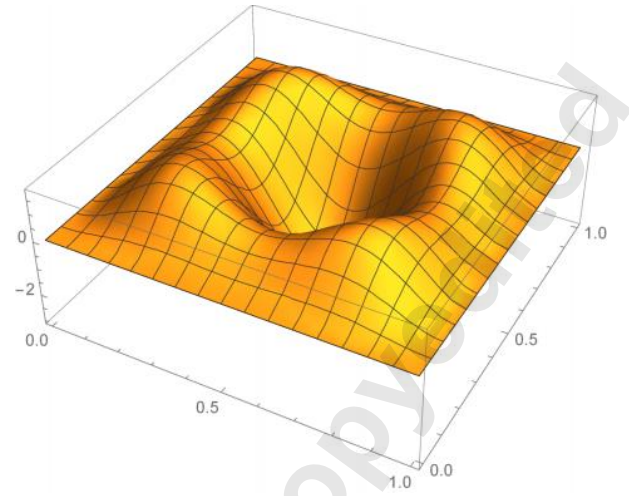

(b)

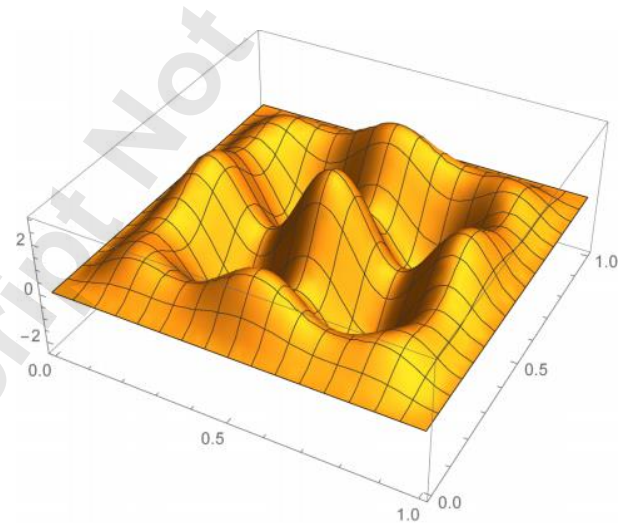

(d)

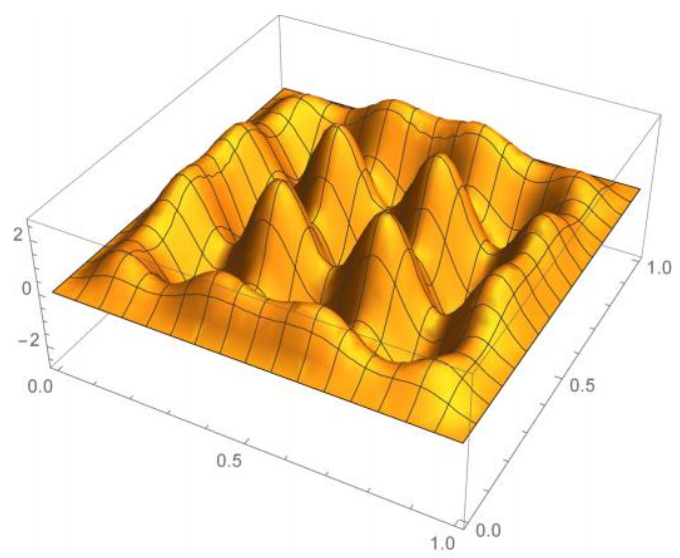

(f)

Fig. 8 The first six symmetric-symmetric transversal modeshapes of a square microplate. 
Table 1 Summary of convergence studies, number of transverse modes required for convergence and the value of the non-dimensional parameter $\alpha_{2} V_{d c}^{2}$ at the pull-in instability.

\begin{tabular}{|l|c|c|}
\hline Model No. & No. of modes for convergence & Voltage parameter $\alpha_{2} V_{d c}^{2}$ \\
\hline Model I & 6 & 188 \\
\hline Model II & 6 & 191 \\
\hline Model III & 4 & 186 \\
\hline Model IV & 4 & 194 \\
\hline Model V & 4 & 192 \\
\hline
\end{tabular}


Table 2 A comparison of the time taken by FE model implemented in COMSOL and the reduced order models to solve the system under an electrostatic DC voltage.

\begin{tabular}{|l|c|}
\hline Model & Time taken (Seconds) \\
\hline FE model & 125 \\
\hline Model II & 0.140 \\
\hline Model III & 0.046 \\
\hline Model IV & 0.046 \\
\hline Model V & 0.031 \\
\hline
\end{tabular}


Table 3 Frequency parameter $\lambda=\omega a^{2} \sqrt{\frac{\rho}{D}}$ for first six symmetric-symmetric modeshapes of a square microplate calculated using the FEM software COMSOL.

\begin{tabular}{|c|c|}
\hline Mode $\left(\phi_{i}\right)$ & Non-dimensional frequency parameter, $(\lambda)$ \\
\hline$\phi_{1}$ & 35.89 \\
\hline$\phi_{2}$ & 130.98 \\
\hline$\phi_{3}$ & 216.54 \\
\hline$\phi_{4}$ & 302.87 \\
\hline$\phi_{5}$ & 383.58 \\
\hline$\phi_{6}$ & 545.7 \\
\hline
\end{tabular}

